\title{
Transposition
}

Musique et Sciences Sociales

1 | 2011

Polyphonie et société

\section{Mondher Ayari et Hamdi Makhlouf (ed.), Musique, Signification et Émotion}

Paris, Delatour, 2010, 408 p.

\section{Theresa Schmitz}

\section{(2) OpenEdition}

\section{Journals}

Édition électronique

URL : http://journals.openedition.org/transposition/406

DOI : $10.4000 /$ transposition.406

ISSN : 2110-6134

\section{Éditeur}

CRAL - Centre de recherche sur les arts et le langage

\section{Référence électronique}

Theresa Schmitz, «Mondher Ayari et Hamdi Makhlouf (ed.), Musique, Signification et Émotion »,

Transposition [En ligne], 1 | 2011, mis en ligne le 01 février 2011, consulté le 22 septembre 2020. URL http://journals.openedition.org/transposition/406 ; DOI : https://doi.org/10.4000/transposition.406

Ce document a été généré automatiquement le 22 septembre 2020.

La revue Transposition est mise à disposition selon les termes de la Licence Creative Commons Attribution - Partage dans les Mêmes Conditions 4.0 International. 


\section{Mondher Ayari et Hamdi Makhlouf (ed.), Musique, Signification et Émotion}

Paris, Delatour, 2010, 408 p.

\section{Theresa Schmitz}

\section{RÉFÉRENCE}

Mondher Ayari et Hamdi Makhlouf (dir.), Musique, Signification et Émotion, Paris, Delatour, 2010, 408 p.

1 «Voici un livre qui vient combler un vide dans la littérature sur la musique ${ }^{1}$ ». Nous reprenons volontairement le constat de Michel Imberty, qui introduit le vaste sujet des émotions auquel s'intéresse l'ouvrage Musique, Signification et Émotion, paru sous la direction de Mondher Ayari et Hamdi Makhlouf.

2 Lors de la présentation publique de l'ouvrage au centre culturel d'Égypte, le 24 juin 2010, ses éditeurs soulignaient qu'ils avaient souhaité à l'origine donner une trace écrite à un cycle de conférences interdisciplinaires sur les trois notions qui forment le fil rouge de l'ouvrage. Mais il ne s'agit pas là d'une simple publication des actes: d'autres chercheurs furent sollicités pour apporter leur contribution à un ouvrage riche et diversifié. Il est vrai que les discussions scientifiques, si enrichissantes et éclairantes soient-elles au cours de conférences, s'ouvrent toujours sur de nouvelles perspectives. Une vingtaine d'auteurs, confirmés ou jeunes chercheurs, ont été invités à exprimer leur point de vue scientifique et personnel sur la relation qui unit musique et émotion. La relation entre les trois notions, musique, signification et émotion, n'est cependant pas thématisée, les textes portant d'une part sur la signification de la musique, d'autre part sur l'émotion musicale.

Le vide évoqué par Michel Imberty renvoie à notre avis surtout à l'absence, jusqu'alors, d'une véritable confrontation d'approches méthodologiques de ces notions. On a déjà essayé, il est vrai, de déchiffrer la relation entre l'émotion humaine et la musique - les musiques -, que cette dernière soit envisagée comme son résultat ou son origine. 
L'intérêt scientifique pour la question s'était jusqu'ici limité aux domaines de la psychologie expérimentale de la musique et de la philosophie. La musicologie s'est davantage intéressée aux problèmes de signification de la musique, l'émotion étant généralement considérée comme un paramètre "non scientifique ", accessoire, voire non significatif : l'analyse a cherché sa légitimité dans la construction de normes et de règles abstraites, empêchant de s'interroger sur la relation intime et immédiate qui unit l'œuvre, son créateur et son récepteur (phénomène bien sûr beaucoup moins chiffrable, beaucoup plus difficile à décrire scientifiquement). Surmonter cet écart construit entre la musique et l'être humain, liés par l'émotion et la signification, a été l'ambition d'approches plus anthropologiques ou ethnologiques de la musique.

4 Le mérite de cet ouvrage est donc de réunir des approches et points de vue diversifiés autour de cette question. C'est aussi ce qui rend difficile la tâche de le présenter. C'est pourquoi nous allons nous intéresser, après avoir présenté un bref aperçu des différents types de contributions qui forment l'ouvrage, aux articles qui par leurs thématiques ont plus particulièrement attiré notre attention.

5 Certains des articles reposant sur des approches philosophiques et psychologiques s'interrogent sur l'émotion en musique en référence à la littérature existante (Kant, Hegel, Wittgenstein, Hanslick, Goodman...) ; certains autres cherchent à ouvrir la voie vers d'autres questionnements, tandis que les derniers s'attèlent à la mise en question de certaines méthodes existantes (voir l'article de François Delalande : « Signification et émotion dans les conduites d'écoute musicale»). Dans le domaine musicologique, l'ouvrage nous propose de discuter les trois notions à l'aide d'études de cas embrassant toutes les époques (Renaissance et époque baroque : l'avènement de la seconda pratica et la modernité d'Aristide Quintilien; période classique: la syntaxe discursive dans certaines œuvres de Beethoven; époque "contemporaine»: les quatuors de Chostakovitch, porteurs d'émotions, ainsi que la notion d'infini chez Sciarrino).

6 Parmi tous ces articles, nous avons choisi de nous intéresser plus particulièrement aux textes de Jean-Marc Chouvel, « Une musique sans émotion? » et de Pierre Michel, «Les musiques dites 'contemporaines' peuvent-elles émouvoir? Réflexions subjectives sur la base d'expériences personnelles». Les deux auteurs interrogent le cliché de l'inaccessibilité et de la rationalité «froide» de la musique contemporaine selon des approches opposées, et donc, en quelque sorte, complémentaires.

Jean-Marc Chouvel propose une tentative d'apologie de cette absence d'émotions dans la création contemporaine, formulée la plupart du temps comme une accusation. Sa démarche est d'autant plus intéressante qu'elle cherche les origines de cette particularité dans l'histoire de la musique: son "effet réfrigérant garanti » serait en réalité une réponse aux courants antérieurs. L'auteur répond à la question de savoir si la musique contemporaine peut émouvoir en remarquant que les compositeurs se sont eux-mêmes posé la question de l'émotion (tout comme leurs prédécesseurs), et qu'ils ont justement cherché à s'émanciper de ce qu'ils considéraient comme une forme de "débauche d'émotions sentimentales ». Étant donnée l'absence de lien immédiatement perceptible par le spectateur entre le geste compositionnel et les émotions (émotions immanentes à l'œuvre, émotions que l'œuvre cherche à communiquer ou encore qui ont déclenché l'acte créatif), Chouvel conclut dans un premier moment qu'il s'agit d'un problème de partage d'émotions: le public, souvent, ne saisit pas ces relations " cachées ». Cependant, Chouvel juge que ce problème de partage (soit l'auditeur est " acculturé » à un style de musique et le "subit », soit il ne le connaît pas et demeure 
hermétique), mis en avant par Klaus Huber, peut être surmonté : «l'art finit toujours par imposer sa vérité dès lors que la dialectique est dépassée dans l'accroissement de la conscience" (p.153). La présentation de cette critique ainsi que la conclusion de l'article dérivent ensuite vers une apologie du « rationalisme ", présenté comme tel par «des contemporains qui écoutent des niaiseries» musicales (p. 155): ces derniers ne font pas l'effort d'apprendre à connaître cette musique, qui, sans contenir elle-même d'émotions, peut bien sûr susciter des émotions chez celui qui la produit et celui qui l'écoute. Le rationalisme ne tue pas l'émotion, et peut être une source d'enrichissement. Sans vouloir contester le point de vue de Jean-Marc Chouvel, il nous semble que sa présentation peut contribuer à exacerber et radicaliser les deux points de vue opposés : de même qu'il est simpliste de nier toute capacité à provoquer des émotions aux créations contemporaines, il est un peu réducteur d'expliquer l'incompréhension face à cette musique par le manque de «culture» du plus grand nombre.

8 Pierre Michel, quant à lui, aborde la question à partir de sa propre expérience d'auditeur de musique contemporaine. Il décrit sa perplexité face à l'antagonisme qui existe entre la sensation de facilité ressentie quand il s'agit de décrire ses émotions à l'écoute de certains classiques des musiques actuelles, et l'absence de cette même sensation face à des œuvres contemporaines «savantes». Il pose notamment la question de l'existence d'une diversité d'écoutes que peut développer un individu, de l'écoute professionnelle d'un musicologue à une écoute plus sensorielle, soumise à la prédisposition physique et psychique dans la réception de l'événement sonore. Ces différents types d'attitude possibles face à la musique, ainsi que les circonstances particulières qui constituent le contexte de l'écoute choisie, mènent naturellement à différents types de jugements. L'exemple donné est celui du musicologue, dont l'écoute professionnelle risque d'empêcher des réactions spontanées face à la musique écoutée, qui seraient celles d'une écoute libre de réflexion analytique. Par ailleurs, l'écoute "moyenne» choisirait d'éviter d'emprunter la voie d'une trop grande réflexion. L'auteur conclut sur ces mots : «Adressons ces pensées aux organisateurs de concerts (...), afin que la diversité des émotions soit préservée du point de vue artistique dans notre société, qu'elle soit un "droit" de toutes et tous, et qu'elle ne soit pas "rongée" par le nivellement du divertissement culturel facile et rentable.» (p.168). On ne peut sans doute qu'être d'accord avec cette assertion, mais il nous semble toutefois qu'à force d'être prononcée, elle est devenue une sorte de cliché produit par les défenseurs de la richesse musicale, et de la musique contemporaine en particulier. L'argument de la diversité suffit-il et satisfait-il encore aujourd'hui?

9 Au sein des nombreuses études ethnomusicologiques - nous renvoyons notamment aux quatre articles sur la musique arabe -, François Picard a choisi de nous donner un aperçu de la vie universitaire, à travers la structure et les contenus du séminaire EthnoMusicologiques de l'Université Paris-Sorbonne (organisé en 2009-2010). Cet article, miroir du séminaire, nous présente quatre «aperçus » de travaux, dont des études doctorales en cours (celles de Jeanne Miramon-Bonhoure, Jessica Roda et Mina Dos Santos) : nous regrettons le peu d'espace qui a été réservé à leurs contributions, qui témoignent encore une fois de la multiplicité des approches possibles de la question, y compris au sein d'un même domaine. L'émotion est appréhendée de manière différente selon qu'elle est véhiculée par une pratique conventionnée (les compétitions des chanteurs siciliens) ou savante (le concept du rasa indien), ou bien quand c'est l'émotion elle-même qui fait naître soit la musique (les lamentations de deuil en Russie) 
soit une «culture » et tradition musicale basée sur le souvenir (les traditions judéoespagnols en France). En conclusion, François Picard - qui rejoint ici Pierre Michel par son recours à l'expérience personnelle -, dresse le constat suivant: l'émotion peut certes être prévisible ( "beauté + qualité + attente = émotion garantie », p. 290), mais il demeure un irréductible degré d'inexplicable, qui peut mener à des bouleversements inoubliables dans la série des belles émotions musicales faites au cours de la vie. Finalement, ce sont sans doute ces approches moins conventionnelles qui sont les plus parlantes, face à un objet si difficile à cerner parce qu'il fait appel surtout et quasi exclusivement au ressenti personnel.

De manière générale, l'hétérogénéité des articles d'un point de vue formel peut être quelque peu déconcertante. Non pas que nous souhaiterions défendre une mentalité rigoureuse, mais il s'avère difficile de saisir l'enjeu général de l'ouvrage, ou bien les présupposés de chacune des contributions. À notre avis, il ne s'agit pas là d'un livre à lire de manière stricte, en commençant par le début, il offre plutôt la liberté de se «balader " d'un sujet à l'autre, ou bien d'aller chercher des approfondissements sur une ou plusieurs questions particulières. C'est un de ses points forts, d'ailleurs. Cependant, l'absence d'homogénéité des références biographiques, sans parler du manque de résumés avant chaque article, peuvent gêner son utilisation. Mentionnons enfin une singularité de l'ouvrage, malheureusement réservée aux initiés (ceux qui ont participé à la publication ou à la présentation au centre culturel d'Égypte) : toutes les illustrations reproduites ont été réalisées par Habib Bida spécialement pour l'ouvrage. L'émotion ressentie par l'auteur, qu'il a essayé de communiquer par son geste créateur, pourra nourrir l'imagination de chaque lecteur. Une petite explication éditoriale aurait suffi pour rendre pleinement honneur à cette touche d'originalité.

\section{NOTES}

1. Ouvrage discuté, p. 1. 